\title{
A COMPARATIVE STUDY ON CLASSIFICATION OF IMAGE SEGMENTATION METHODS WITH A FOCUS ON GRAPH BASED TECHNIQUES
}

\author{
Basavaprasad B ${ }^{1}$, Ravi $\mathbf{M}^{2}$ \\ ${ }^{I}$ Asst. Professor, Department of Computer Science, Govt. First Grade College, Raichur, Karnataka, India \\ ${ }^{2}$ Asst. Professor, Department of Computer Science, Govt. First Grade College, Manvi, Raichur, Karnataka, India
}

\begin{abstract}
Image segmentation is the most precarious functions in image processing and analysis. Basically segmentation consequences influence all the subsequent processes of image analysis such as object description and illustration, characteristic dimension, and even the subsequent higher level tasks such as classification of object. Hence, image segmentation is the most important and critical process for assisting the, depiction, delineation and visualization of regions of interest in any image. Physical segmentation of an image is not only a tiresome and time consuming process, but also not exceptionally accurate particularly with the increasing imaging modalities and uncontrollable quantity of images that need to be observed. Therefore it becomes essential to examination current methodologies of image segmentation using computerized algorithms that are precise and entail as little user interaction as probable in particular for medical images. In the image segmentation process, the anatomical organization or the region of interest needs to be defined and extracted out so that it can be viewed independently. In this comparative paper we venture the significant place of segmentation of images in pulling out information for decision making. Afterwards we present the most relative and general methods which we have classified into three categories, first category represents pixel based, second edge based and third represents region based methods in particular graph based methods, highlighting the weaknesses and strengths according to appropriateness for image segmentation applications.
\end{abstract}

Keywords: Classification, Segmentation, Techniques, Graph based, Pixel-based, Edge-based, Histogram, Thresholding, Clusters, Comparative.

\section{INTRODUCTION}

Generally, image segmentation can be defined as a procedure for partitioning a digital image into multiple regions (set of pixels). Image segmentation separates an image into regions with no overlapping. Each region possesses different features including color, intensity, texture or other statistical properties. The main aim of image segmentation is to simplify and transform the representation of an image into something that is further meaningful and simpler to analyze. The result of image segmentation is either a set of regions that together cover up the entire image or a set of contours extracted from the image [1]. From the foregoing general definition, a more specific definition can be given; the process of delineating and separating the anatomical structure (region of interest) so that it can be viewed individually in order to achieve important objectives such as patient diagnosis, radiotherapy planning, and advanced surgical planning and research. Therefore, TRUS is more popular for screening and biopsy guidance for prostate cancer. It is a safe means of imaging the human body because it emits no ionizing radiation, and it uses sound waves of low-power. It is comparatively contemptible and transportable to handle. If the domain of the image is known by I, next, the image segmentation problem is to determine the sets whose union is the complete image I. Therefore, the sets that build up image segmentation must satisfy:

$$
I=U^{k} S_{k}
$$

Where $K=1$.

Where $S_{k} \cap S_{j}=\emptyset, \forall k \neq j$ and each $S_{k}$ is a connected region. Ideally, segmentation method defines those sets that belong to distinctive anatomical structures or regions of interest in the image [4]. One direct way to segment an image is by applying an edge detection technique, especially If the image consists of clear objects on a different intensity background. However, this method can fail, if the image contains noise. It is usually necessary to conduct validation experiments so as to quantify the performance of a segmentation technique. It can be done by comparing the automated segmentation with the manual segmentation of an 
expert. An alternative is using physical phantoms or computational Phantoms. There are numerous image segmentation techniques. However, there is no single or standard segmentation technique that works well for all images. In this paper we have presented a systematic study on image segmentation techniques which we classified into three categories. Firstly pixel-based methods which are explained in section 2, secondly edge-based methods presented in section 3 and finally graph-based methods are presented in Section 4.

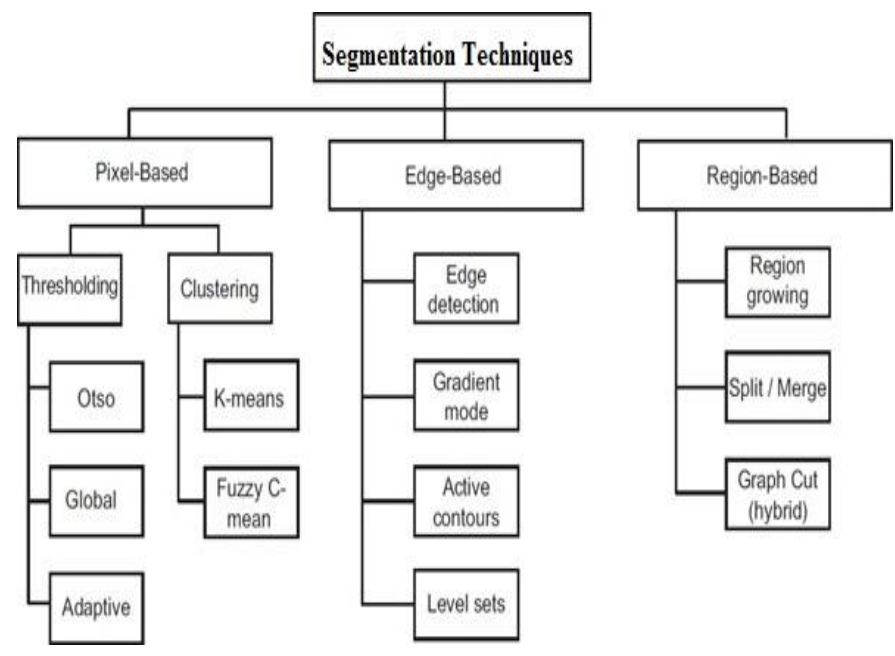

Fig 1: Image Segmentation Techniques

The division of an image into meaningful structures, image segmentation, is often an essential step in object representation, image analysis, apparition, and many other image processing tasks. We focused on how to analyze and correspond to an object, except our assumption the sets of pixels that identified that object was known in advance. In this section, we will focus on methods that find the particular pixels that make up an object. A great variety of segmentation methods has been proposed in the past decades, and some categorization is necessary to present the methods properly here. A disjunction categorization does not seem to be possible though, because even two very different segmentation approaches may share properties that defy singular categorization 1 . The categorization presented in this chapter is therefore rather a categorization regarding the emphasis of an approach than a strict division. The following categories are used:

- Pixel based segmentation is the process of partitioning a digital image into multiple segments or groups of pixels, also known as super pixels.

- Threshold based segmentation, Histogram thresholding and slicing techniques are used to segment the image. They may be applied on an image directly, but also can be combined with preprocessing and post-processing techniques.

- Edge based segmentation. With this method, the edges which are detected in an image are assumed to represent object boundaries, and used to identify these objects.
- Region based segmentation. Where an edge based technique may attempt to find the object boundaries and then locate the object itself by fulfilling them in, a region based method takes the opposite approach Structural Segmentation Methods

These methods utilize information about the structural features of the image to implement segmentation of the target image. Some of the common structural methods include edgedetection, graph searching, deformable models, is on surface and level set. We highlight some.

\section{PIXEL BASED IMAGE SEGMENTATION}

\subsection{Thresholding Methods:}

We have taken thresholding method as an example to explain pixel based image segmentation. The thresholding technique is the simplest image segmentation technique. It is based on the assumption that the objects and the background in the image have a bimodal distribution. Typically, these assumptions not valid for most images, especially medical ones. The key point in this segmentation technique is to determine an intensity rate, which is called the threshold, which separates the desired classes. The main advantages of deformable models are their ability to directly generate closed parametric curves or surfaces from images and their incorporation of a smoothness constraint that provides robustness to noise and spurious edges. Moreover, they offer sub-pixel accuracy for the boundary representation, which may be important to medical applications. An important drawback of the snakes is that they require manual interaction to place an initial model or to choose appropriate initial parameters. Besides, they cannot be used in non-interactive applications, unless they are initialized close to the structure to be segmented because they are designed as interactive models. Thresholding segmentation transforms input image, I, to a binary image, $\mathrm{g}$, by grouping the pixels with intensities, higher than the threshold into one the class, and the further pixels into another class. For any 2D digital image [16],

$I(i, j)$ for $i=1,2, \ldots, M$ and
$j=1,2, \ldots, N$

The technique of thresholding is defined as:

$g(i, j)=\left\{\begin{array}{l}1 \text { for } I(i, j) \geq T \\ 0 \text { for } I(i, j) \geq T\end{array}\right.$

Some images can be segmented by using more than one point of thresholding, also called as multi-thresholding, as, where $T_{1}$ and $T_{2}$ are the two thresholds segmenting the image. The output image, resulting from multi-thresholding, is no longer binary, but consists of a limited number of grey levels as 
follows:

$$
g(i, j)=\left\{\begin{array}{c}
1 \text { for } I(i, j) \geq T_{1} \\
0 \text { for } I(i, j) \geq T_{2} \\
N \text { for } T_{m} I(i, j) \mathrm{T} \mathrm{Ng}-1
\end{array} \ldots\right.
$$

$\mathrm{Ng}$ is the image grey levels number in the image (256).

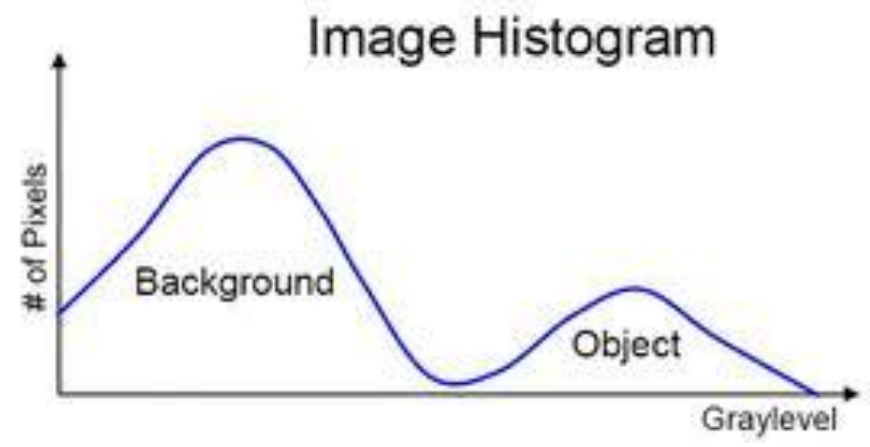

Fig 2: Histogram showing three apparent classes using two thresholds $T_{1}$ and $T_{2}$.

\section{EDGE BASED IMAGE SEGMENTATION}

The problem of detecting edges in an image is a basic function in analysis of images. It engages characterizing the object boundaries in an image using edge information. It then makes edge detection very handy for segmentation. Edge detection techniques are commonly used for detecting edges in an image to perform segmentation process. The traditional edge detection methods usually use the edge detection operators, which are based mainly on the gradation such as Sobel, Robert, and Prewitt edge detectors . Usually edges occur at the point of intersection of two regions with intensities which are varying. The advantage of these techniques is that they work very well only on images with high-quality disparity between different regions. Their disadvantages include; they detect all the edges; hence, it is very difficult to find the relation between the edges and the region of interest. In addition, those algorithms are sensitive to noise. Edge-based segmentation corresponds to a huge group of methods based on information concerning to edges in an image.

- Edge-based segmentations depend on edges found in an image by the edge detecting operators. These edges spot image locations of discontinuities in color, texture, gray level etc.

- Image resulting from the method of edge detection cannot be used as a result of segmentation. Accompanying processing steps must go after to merge edges into edge chains that correspond to improve with borders in the image.

- The final desire is to reach at least a fractional segmentation; that is, to bunching neighboring edges into an image where only edge chains with an association to existing things or image parts are in attendance.
- The more aforementioned information that is available to the segmentation procedure, the better the image segmentation results that can be attained.

- The most general problems of edge-based image segmentation are an edge occurrence in locations anywhere with no border and no edge exists where an actual border is there.

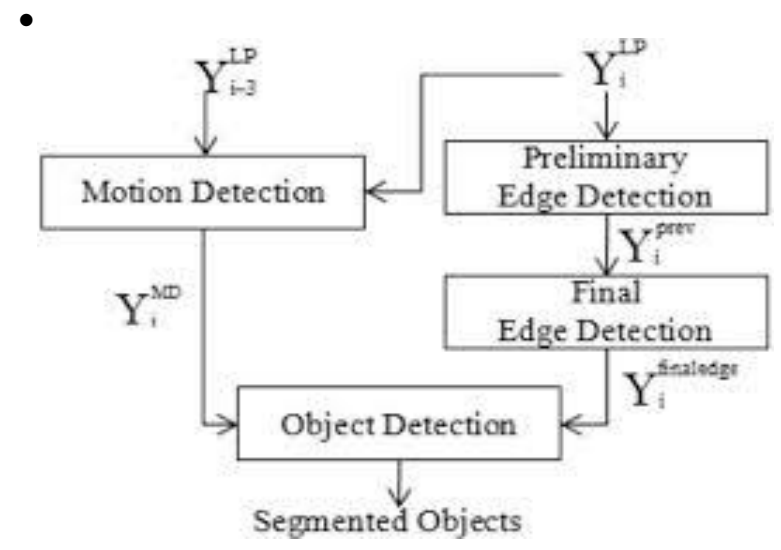

Fig 3: Edge based image segmentation flow.

\section{REGION BASED METHODS}

\subsection{Region Growing Methods}

We have taken region growing method as an example to explain region based image segmentation. Region growing takes advantage of an important fact that the pixels close together have similar gray values. The main idea of this approach consists in the observation that the pixels belonging to one element of the object can possess similar properties, for example, the gray value. Therefore if the considered pixel has gray value that is near the common gray value of the region, this pixel can be associated into this region. This technique generally consists of the following three steps; Initially, a new region consists of only one pixel which is called the seed pixel is selected. This pixel is determined by user, and issued as apriori information. The given initial pixel determines the8neighbour where all pixels are sequentially checked. If a pixel does not belong to any other regions it can be associated into the given region if the difference between the gray value of the pixel and the averaged gray value of the whole region is less than a predefined threshold, T. Second, when the pixel is connected to the region the averaged gray value of this region is re-calculated. After all the neighboring pixels are reviewed, new neighborhood for the enlarged region will be determined and the first step is.

\subsection{Graph Search Algorithm:}

Graphs are an abstract representation consisting of a set of vertices $V=\{v 1, \ldots, v n\}$ and a set of edges $E=\left\{\ldots,\left\{v_{i}, v_{j}\right\}, \ldots\right\}$ where $\quad \mathrm{k}=\left\{v_{i}, v_{j}\right\}$ indicates that there is an edge ( $\operatorname{arc})$ from vertex $i$ to vertex 
j. Each edge has an associated weight representing the cost of transition from $v_{i}, v_{j}$ as shown in Fig. In these algorithms, edges and surfaces are represented as graphs, and the algorithm tries to find the lowest-cost path between two nodes of the graph using any search algorithm. Those algorithms have the advantage that they can perform well even if the separations between regions are broken. Their disadvantage is that of requiring surfaces to be represented as graphs, which could be tricky [1]. These graph based algorithms are playing a vital role in image segmentation especially in medical image processing which is topic emerging as most active research from the past two decades [8].

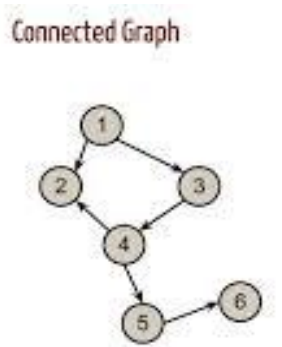

Connected

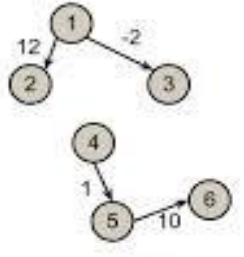

Disconnected
Fig 4: Both Connected and disconnected graphs.

\section{RESULTS}

The result of this is paper is mentioned in the below images and table.

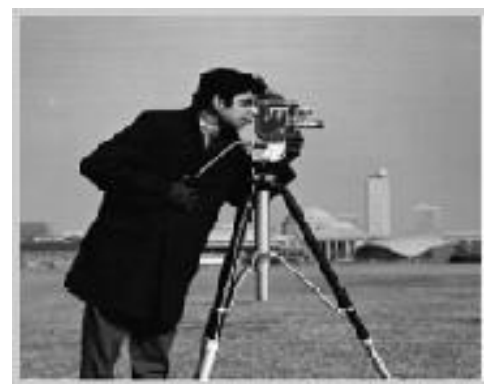

a) Original Cameramen Image

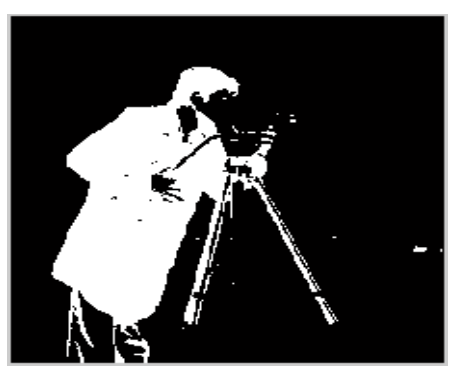

b) Pixel Based Image Segmentation

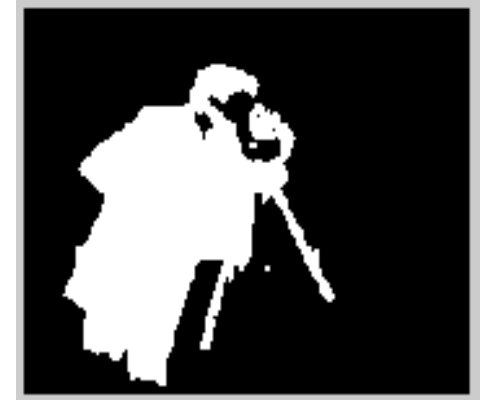

c)Edge Based Image Segmentation

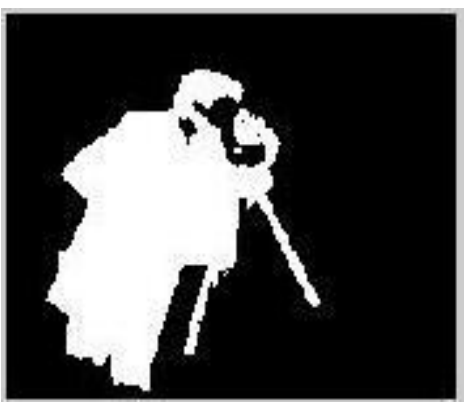

d) Graph Based Image Segmentation

Fig 5: Comparative Segmented Images

Table 1: Comparison of classified methods

\begin{tabular}{|l|l|l|}
\hline Method & Advantage & $\begin{array}{l}\text { Segmentation } \\
\text { effect }\end{array}$ \\
\hline $\begin{array}{l}\text { Pixel Based } \\
\text { Segmentation }\end{array}$ & More Complexity & Normal \\
\hline $\begin{array}{l}\text { Edge Based } \\
\text { Segmentation }\end{array}$ & $\begin{array}{l}\text { Average } \\
\text { Complexity }\end{array}$ & Average \\
\hline $\begin{array}{l}\text { Graph Based } \\
\text { Segmentation }\end{array}$ & $\begin{array}{l}\text { Very low } \\
\text { complexity }\end{array}$ & Good \\
\hline
\end{tabular}

\section{CONCLUSIONS}

We have thoroughly discussed the useful methods of pixel based, edge based and region based (graph) methods of image segmentation. This systematic comparison study is helpful for individual researchers to do research in the ground of image segmentation. These Image segmentation techniques are extremely efficient particularly, graph based image segmentation methods which comes under the third category i.e., region based methods. Medical image processing is one of the main dynamic research topics in image processing [18]. Most modern research in image segmentation has tinted the potential of graph based techniques for medical applications. In order to implement graph theory in image segmentation professionally in particular in medical image processing we need to set up implementation between mathematical outstanding junior scientists and biological scientists, and 
describe the sketch to build up the new tools on this domain. The motivation should be on the study of properties of Euler graphs, minimal spanning trees, Fuzzy graphs, shortest paths trees, minimal cuts and Normalized cuts and we re-examine these ideas for image segmentation purposes. From this comparison study we conclude that there is no universal segmentation method that can be executed for all types of images, but a number of techniques do healthier than others for particular types of images representative better performance can be attained by selecting appropriate algorithm or combination of suitable techniques [29].

\section{REFERENCES}

[1]. Basavaprasad B. and Ravindra S. Hegadi, "A Survey on Traditional and Graph Theoretical Techniques for Image Segmentation", Recent Advances in Information Technology, International Journal of Computer Applications, Pages: 38-46, 2014.

[2]. Shanbehzadeh J. and Rastgarpour M., "Application of AI Techniques in Medical Image Segmentation and Novel Categorization of Available Methods and Tools", International Multi-Conference of Engineers and Computer Scientists Proceedings, Volume: 1, Hong Kong, 2011.

[3]. Zhang, Y. J, "An Overview of Image and Video Segmentation in the last 40 years", 6th International Symposium on Signal Processing and Its Applications Proceedings, Pages: 144-151, 2001.

[4]. Wahba Marian, "Automated Modified Region Growing Technique for Prostate Segmentation in Trans-Rectal Ultrasound Images", Master's Thesis, Department of Electrical and Computer Engineering, University of Waterloo, Waterloo, Ontario, Canada, 2008.

[5]. Shapiro L. G. and Stockman G. C., Computer Vision, Prentice-Hall Publications, New Jersey, 2001.

[6]. Pham D. L, Prince J. L. and Xu C, "Current Methods in Medical Image Segmentation", Biomedical Engineering, Annual Review, Volume: 2, Pages: 315-38, 2000.

[7]. Lakare S., and Kaufman A., "3D Segmentation Techniques for Medical Volumes", A Center for Visual Computing", Department of Computer Science, State University of New York, December 2000.

[8]. Viergever M. A and Lobregt S., "A Discrete Dynamic Contour Model", Medical Imaging, an IEEE Transactions, Volume: 14, Pages: 12-24, 1995.

[9]. Kass M., Terzopoulos D. and Witkin A., "Snakes: Active Contour Models", IJCV, Volume: 1, Pages: 321-331, 1988.

[10]. Szeliski R. and Terzopoulos D., "Tracking with Kalman Snakes", Active Vision and Artificial Intelligence, Pages: 320, Cambridge, the MIT Press, 1992.

[11]. Hamarneh G., "Towards Intelligent Deformable Models for Medical Image Analysis", Ph.D. Thesis, Department signals and Systems, Electrical and Computer Engineering School, Technology of Chalmers University, 2001.

[12]. Awad, J.G.E., "Prostate Segmentation and Regions of Interest Detection in Transrectal Images of Ultrasound",
Thesis of Doctor of Philosophy, University of Waterloo, Waterloo, Ontarion, Canada, 2007.

[13]. Sahoo P. K., Farag A. A., and Yeap Y. P., Threshold Selection Based on Histogram Modeling, IEEE International Conference on Systems, Man and Cybernetics, Volume: 1, Pages: 351-356, 1992.

[14]. Pathak S. D., Grimm P. D., Chalana V., and Kim Y.,Pubic Arch Detection in Transrectal Ultrasound Guided Prostate Cancer Therapy, IEEE Transactions on Medical Imaging, Volume: 17, Pages: 762-771, 1998.

[15]. Potocnik B., Solina F., and Zazula D., "Classical Image Processing vs. Computer Vision Techniques in ComputerAssisted and Automated Detection of Follicles in Ultrasound Images of Ovary", Image Processing and its Applications, 6th International Conference, Volume: 2, Pages: 551-555, 1997.

[16]. Lee C., Huh S., Ketter T. A., and Unser M., "Unsupervised Connectivity-Based Thresholding Segmentation of Midsagittal Brain MR Images, Computers in Biology and Medicine, Volume: 28, Pages: 309-338, 1998.

[17]. Schalkoff R.J., Pattern Recognition: "Statistical, Structural and Neural Methods", John Wiley and Sons, 1992.

[18]. Pham D. L., Prince J. L. and Xu C., "Survey of present Methods in Medical Image Segmentation", Annual Review of Engineering (Biomedical), 1998.

[19]. Xu R., and Wunsch D., "A Survey of Clustering Algorithms", Neural Networks IEEE Transaction, Volume: 16, May 2005.

[20]. Hebert T. J., "Quick Iterative Segmentation of High Resolution Medical Images", Nuclear Science, IEEE Transaction, Volume: 44, Pages: 1363-1367, 1997.

[21]. Hall L.O., Bensaid A.M., Bezdek J.C. Clarke L.P., Velthuizen R.P.Silbiger M.S., "A Comparison of Neural Network and Fuzzy Clustering Techniques in Segmenting Magnetic Resonance Images of the Brain", IEEE Transactions on Neural Networks, Volume: 3, Pages: 672 $682,1992$.

[22]. Gelenbe E., Krishnan K.R.R. and Feng Y., "A Neural Network Methods for Volumetric Magnetic Resonance Imaging of the Human Brain", IEEE Proceedings, Volume: 84, Pages: 1488-1496, 1996.

[23]. Hall L.O., Clarke L.P. and Bezdek J.C., "Review of MR Image Segmentation Techniques Using Pattern Recognition", Medical Phys, Volume: 20, Pages: 1033-1048, 1993.

[24]. Reddick W.E., Cook E.N., Glass J.O., Elkin T.D., and Deaton R.J., "An Automated Segmentation and Classification of Multispectral Magnetic Resonance Images of Brain Using Artificial Neural Networks", IEEE Transactions on Medical Imaging, Volume: 16, Pages: 911-918, 1997.

[25]. Vilarino D.L., Cabello D., Brea V.M. and Pardo J.M., "Discrete-Time CNN for Image Segmentation by Active Contours", Proceedings of Pattern Recognition, Volume: 19, Pages: 721-734, 1998.

[26]. Tournoux $P$ and Talairach J. "Co-Planar Stereotaxic Atlas of the Human Brain", Proportional System on 3D: An Approach to Cerebral Imaging, Thieme Medical Publisher, 1988. 
[27]. Sandor S., and Leahy R., "Surface-Based Labeling of Cortical Anatomy Using a Deformable Atlas", Medical Imaging, IEEE Transactions, Volume: 16, Pages: 41-54, 1997.

[28]. Davatzikos C., Vaillant, M., Resnick S., Prince J. L., Letovsky S and Bryan R. N., "A Computerized Method for Morphological Analysis of the Corpus Callosum", An International Journal of Computer Application, Volume: 20, Pages: 88-97, 1996.

[29]. Basavaprasad B. and Ravindra S. Hegadi, "An Improved GrabCut Technique for segmentation of Color Images", Recent Advances in Information Technology, an International Journal of Computer Applications, Pages: 5-8, 2014.

[30]. Garcia-Consuegra J., Cisneros G. and Navarro E., "A Sequential Echo Algorithm Based on the Integration of Clustering and Region Growing methods", Proceedings of IGARSS, Volume: 2, Pages: 648-650, 2000.

[31]. Pan W., "Application of Region Growing Algorithm on Handwritten Numerical Recognition", thesis for Master of Science, Department of Computer Science and Engineering, Tatung University, 2004. 